\title{
A Carrier Parameter Decoupling Algorithm for High Order APSK Modulation
}

\author{
Xiangchuan Gao, Xupeng Zhang $\mathbb{D}^{D}$, Linlin Duan, Kexian Gong, Peng Sun, Wei Wang $\mathbb{D}$, \\ Hua Jiang, and Zhongyong Wang
}

School of Information Engineering, Zhengzhou University, Zhengzhou 450000, China

Correspondence should be addressed to Wei Wang; iewwang@zzu.edu.cn

Received 15 August 2021; Revised 18 October 2021; Accepted 26 November 2021; Published 16 December 2021

Academic Editor: Omprakash Kaiwartya

Copyright (C) 2021 Xiangchuan Gao et al. This is an open access article distributed under the Creative Commons Attribution License, which permits unrestricted use, distribution, and reproduction in any medium, provided the original work is properly cited.

\begin{abstract}
In satellite communication, carrier parameter estimation usually uses a serial structure, and the accuracy of frequency offset estimation (FOE) will greatly affect the accuracy of phase offset estimation (POE). A new carrier synchronization mode (NCSM) can realize the decoupling of carrier FOE and POE to a certain extent, but this mode is based on multibase phase shift keying (MPSK) modulation analysis, the decoupling performance is poor when uses in amplitude phase shift keying (APSK) modulation, and the decoupling performance of NCSM has a low tolerance of frequency offset. An improved carrier parameter estimation decoupling technique is proposed to solve these problems. The simulation results show that, compared with the original method, under the premise of ensuring the accuracy of carrier parameter estimation, the proposed method is more robust to the modulation mode, the POE has stronger antioffset ability, and the normalized FOE range has been significantly enhanced.
\end{abstract}

\section{Introduction}

With the increasing demand of satellite communication system, satellite communications are also more closely linked to fifth generation (5G) wireless networks. On the one hand, security is an important factor in wireless communication systems $[1,2]$. On the other hand, high-speed and reliable wireless communication is a hot research topic in academia and industry, and various related researches have been carried out for different communication scenarios [3]. In [4], the authors qualitatively analyse the research progress of geocast routing (GR) in intelligent transportation systems and also analyse some future research challenges of GR. In [5], the authors proposed a predictive distributed cluster mechanism that has further improved the transmission performance of wireless sensor network devices. In order to further improve the transmission performance of satellite communication, the role of high-order modulation in satellite communication is becoming more and more important. Compared with
QAM modulation signal, MAPSK modulation signal is widely used in satellite communication because it is more suitable for nonlinear communication channels. The latest generation of digital video broadcasting standards (Digital Video Broadcasting-Second Generation Extensions, DVBS2X) adds a variety of high-order APSK modulations on the basis of the original modulation mode, among which the highest order of APSK is up to 256 [6]. Due to the existence of multiple amplitudes and narrower phase discrimination of APSK signals, the synchronization algorithm of high-order modulated signals is challenging, and there are urgent needs to design advanced synchronization algorithm for high-order modulation signals in satellite communication.

In satellite communication, carrier frequency error of receiver mainly comes from Doppler frequency shift and carrier frequency difference introduced by the crystal oscillator error of receiver $[7,8]$. At the same time, due to the sudden nature of satellite communication, pilots are usually introduced to help achieve fast acquisition and FOE, but 
the limited spectrum resources limit the pilot frequency overhead. Therefore, the large Doppler shift and pilot cost are the two main factors affecting the performance of coherent demodulation of satellite communication. Aiming at the above problems, various carrier synchronization algorithms have been proposed in many literatures. Classical forward frequency offset estimation includes algorithms such as M\&M [9], L\&R [10], and Fitz [11]. These estimation algorithms can only approach the Cramer Rao Lower Bound (CRLB) at high SNR, and it is difficult to take into account both the accuracy of FOE and the range of FOE. In [12, 13], the authors propose a maximum likelihood iterative synchronization based on the expected maximum algorithm. By combining carrier synchronization with the decoder, accurate synchronization could be obtained, but the synchronization range cannot be guaranteed and the complexity is impractical. In [14], the authors propose a code-assisted carrier synchronization method based on frequency search, whose complexity is less than the original EM algorithm. Although the abovementioned coding-assisted carrier synchronization algorithms can work at low signal-to-noise ratios, the scope of application of such algorithms is limited. In [15], the authors propose a fast Fourier transform (FFT) frequency offset estimation algorithm based on interpolation and binary search. In [16], an improved Discrete Fourier Transform (DFT) frequency offset estimation algorithm is proposed, which solves the problem of the limited estimation range of Candan algorithm. In [17], the authors use coarse estimation based on discrete Fourier transform and fine estimation based on golden section search algorithm to improve the performance of frequency offset estimation. Although the performances of the abovementioned FFTbased frequency offset estimation algorithm are quite good, the complexity of this type of algorithm is still high. In order to reduce complexity, the authors in [18] proposed the maximum likelihood carrier FOE method based on the equalinterval pilot symbol, leading to higher estimation accuracy and lower SNR threshold, nevertheless, the synchronization range is still quite small. In [19], the carrier synchronization is carried out by structure of autocorrelation plus crosscorrelation to achieve high accuracy and wide estimation range. Unfortunately, the estimated performance becomes worse when the pilot interval is large. In $[20,21]$, the authors consider the case that the first sampling time of the receiver is placed in the middle of the burst structure and gives the Cramer-Rao Bound (CRB) for the joint FOE of this case and the traditional case, respectively. However, in practical application, it is impossible to set the sampling zero time position artificially. Moreover, the possible influence of this structure on carrier synchronization performance has not been further discussed in [20,21]. The authors in [22] proposed a frequency phase decoupling technique based on autocorrelation operator under MPSK modulation. However, the direct transplant to APSK or other high-order modulation signals will lead to poor performance.

To solve the above issues, this paper proposes a new carrier phase decoupling technique for high-order APSK signals. We firstly obtain the decoupling factor by the product of the signal of modulation removal and the autocorrelation function. Then, we add a phase unwrapping module to overcome the influence of high FO on the decoupling factor. Using the above decoupling factor, we change the order of arg operator and summation to get the phase offset estimation, eliminating the influence of amplitude of modulated signal to POE. Simultaneously, we also carry out FOE with the autocorrelation function. The simulation results demonstrate that the decoupling performance of the proposed method is better than NCSM, and the FOE has high estimation accuracy and large estimation range.

\section{Signal Model}

For the convenience of readers, we list and explain all the symbols used in this paper in Table 1.

In a Gaussian channel, the baseband discrete signal after ideal timing synchronization can be expressed as

$$
r(k)=c(k) e^{j\left(2 \pi f_{d} T k+\theta\right)}+n(k), k \in \kappa,
$$

where $c(k)$ is known modulated signal, $f_{d}$ and $\theta$ are frequency offset (FO) and phase offset (PO) generated during signal reception, $T$ is the symbol period, $n(k)$ is the complex Gaussian random variable, its mean is zero, and its variance is $\sigma=N_{0} / 2 . \kappa \triangleq\{N, N+1, \cdots, N+L-1\}$ is sampling time set corresponding to the pilot symbol in the data frame, and $|\kappa|=L$. The data frame structure is shown in Figure 1, where $L$ is pilot length, and $N$ and $\widehat{N}$ are the pilot start position and the total length of the "data-pilot" structure.

We multiply both sides of (1) by $c^{*}(k)$ to remove the modulation information of the received signal, yielding

$$
z(k)=r(k) c^{*}(k)=a(k) e^{j\left(2 \pi f_{d} T k+\theta\right)}+d(k),
$$

where $z(k)$ is called unmodulated information signal, $d$ $(k)=c^{*}(k) n(k)$ is Gaussian white noise, and $a(k)=c(k) c^{*}($ $k)$ is a real constant.

The principle block diagram of the traditional carrier synchronization mode (TCSM) is shown in Figure 2. First, the pilot information is used to estimate the FO, then, the result of FOE is compensated to the demodulation signal, and finally, the POE is performed. In this mode, the accuracy of the FOE will affect the backward POE. In order to ensure the accuracy of the FOE, more pilot overhead is usually needed. Therefore, this processing method is not applicable to the communication environment with limited spectrum resources.

In [22], the authors proposed the NCSM suitable for MPSK, and it can realize the decoupling of FO and PO to a certain extent. The structure diagram of NCSM is shown in Figure 3.

The autocorrelation operator formula based on the demodulation signal can be obtained from (2) (for MPSK signals, $|a(k)|=1)$.

$$
R(\alpha)=\frac{1}{L-\alpha} \sum_{k=N}^{N+L-\alpha-1} z^{*}(k) z(k+\alpha)=e^{j 2 \pi f_{d} T \alpha}+\psi(\alpha),
$$


TABLE 1: Symbol description.

\begin{tabular}{lc}
\hline$r(k)$ & Received signal \\
$c(k)$ & Known modulated signal \\
$f_{d}$ & Frequency offset \\
$\theta$ & Phase offset \\
$\widehat{f}_{d}$ & Estimated frequency offset \\
$\widehat{\theta}$ & Estimated phase offset \\
$n(k)$ & Complex Gaussian random variable \\
$T$ & Symbol period \\
$N$ & Pilot start position \\
$\widehat{N}$ & Total length of data \\
$z(k)$ & Unmodulated information signal \\
$d(k)$ & Gaussian white noise \\
$a(k)$ & Signal energy \\
$R(\alpha)$ & Autocorrelation operator \\
$D(k)$ & Decoupling factor \\
$\psi(a)$ & Noise interference term \\
$\Psi(\alpha)$ & Noise interference term \\
$\widehat{\Psi}(k)$ & Noise interference term \\
$Z_{s}(k)$ & Phase angles of $z(k)$ \\
$R_{s}(\alpha)$ & Phase angles of $R(\alpha)$ \\
\hline &
\end{tabular}

where $\psi(a)$ is the sum of noise terms

$$
\begin{aligned}
\psi(\alpha)= & \frac{1}{L-\alpha} \sum_{k=0}^{L-\alpha-1} e^{-j 2 \pi f_{d} T(k+N)} d(k+\alpha+N) \\
& +e^{j 2 \pi f_{d} T(k+\alpha+N)} d^{*}(k+N)+d(k+\alpha+N) d^{*}(k+N) .
\end{aligned}
$$

The decoupling factor $D(k)$ is expressed as

$$
D(k)=\left.z(k) \cdot R^{*}(\tilde{\alpha})\right|_{\tilde{\alpha}=L-1 / 2}=\left.z(k) \cdot\left[e^{-j 2 \pi f_{d} T \tilde{\alpha}}+\psi^{*}(\tilde{\alpha})\right]\right|_{\tilde{\alpha}=L-1 / 2}
$$

Then, the decoupling factor $D(k)$ of carrier parameter estimation is sent to the PO estimator based on maximum likelihood criterion to obtain the $\mathrm{PO}$

$$
\begin{aligned}
\widehat{\theta} & =\arg \left\{\sum_{k=N}^{N+L-1} D(k)\right\}=\arg \left\{\mathrm{e}^{j \theta} \mathrm{e}^{j 2 \pi f_{d} T N} \sum_{k=0}^{L-1} \mathrm{e}^{j 2 \pi f_{d} T(k-L-1 / 2)}+\bar{\psi}\right\} \\
& =\arg \left\{\mathrm{e}^{j \theta} \mathrm{e}^{j 2 \pi f_{d} T N} \frac{\sin \left(\pi f_{d} T L\right)}{\sin \left(\pi f_{d} T\right)}+\bar{\psi}\right\} .
\end{aligned}
$$

When the signal-to-noise ratio (SNR) of the received sig- nal is high, we get $|\bar{\psi}| \approx 0$, then, $\widehat{\theta}$ is approximately

$$
\widehat{\theta} \approx \begin{cases}\theta, & N=0 \text { and } \frac{\left|f_{d} T\right| \leq 1}{L}, \\ H_{1}\left(\theta, f_{d}, N, L\right), & N \neq 0 \text { or } \frac{\left|f_{d} T\right|>1}{L},\end{cases}
$$

where $H_{1}\left(\theta, f_{d}, N, L\right)$ is a function representing phase ambiguity, and its value is related to the $\mathrm{PO}$, the $\mathrm{FO}$, the pilot initial position, and the pilot length. We can see from (7) that when the FO is small, the estimated value of PO is still approximately equal to the true value. However, when the received signal is APSK, the derivation of (6) is not valid. In order to make the decoupling method suitable for different modulation modes, we improved NCSM and proposed an improved carrier parameter estimation decoupling technique (ICPEDT). The specific structure is introduced in next section.

\section{ICPEDT Based on Parameter Correcting}

Figure 4 is the block diagram of ICPEDT. Compared to NCSM, the ICPEDT changed the function of the comparator and added unwrapping operator. The specific analysis is as follows.

For APSK modulation, the $|a(k)| \neq 1$ in (2), so the autocorrelation operator formula of demodulation signal is changed as

$$
R(\alpha)=\frac{1}{L-\alpha} \sum_{k=N}^{N+L-\alpha-1} z^{*}(k) z(k+\alpha)=\gamma_{\alpha} e^{j 2 \pi f_{d} T \alpha}+\Psi(\alpha)
$$
term

where $\gamma_{\alpha}=a^{*}(k) a(k+\alpha), \Psi(\alpha)$ is noise interference

$$
\begin{aligned}
\Psi(\alpha)= & \frac{1}{L-\alpha} \sum_{k=0}^{L-\alpha-1} \underbrace{c^{2}(k+N) c(k+\alpha+N)}_{\lambda_{1}(\alpha)} e^{-j 2 \pi f_{d} T(k+N)} n(k+\alpha+N) \\
& +\underbrace{c(k+N) c^{2}(k+\alpha+N)}_{\lambda_{2}(\alpha)} e^{j 2 \pi f_{d} T(k+\alpha+N)} n^{*}(k+N) \\
& +\underbrace{c(k+\alpha+N) c(k+N)}_{\lambda_{3}(\alpha)} n(k+\alpha+N) n^{*}(k+N) .
\end{aligned}
$$

The $D(k)$ can be expressed as

$$
D(k)=\left.z(k) \cdot R^{*}(\tilde{\alpha})\right|_{\tilde{\alpha}=L-1 / 2}=z(k) \cdot\left[\gamma_{\frac{L-1}{2}} e^{-j 2 \pi f_{d} T L-1 / 2}+\Psi^{*}\left(\frac{L-1}{2}\right)\right] .
$$

The expression of POE is

$$
\widehat{\theta}=\frac{1}{L} \sum_{k=N}^{N+L-1} \arg \{D(k)\}=\frac{1}{L} \sum_{k=N}^{N+L-1} \arg \left\{a_{k} \gamma_{L-1 / 2} \mathrm{e}^{j\left(2 \pi f_{d} T(k-L-1 / 2)+\theta\right)}+\widehat{\Psi}(k)\right\},
$$




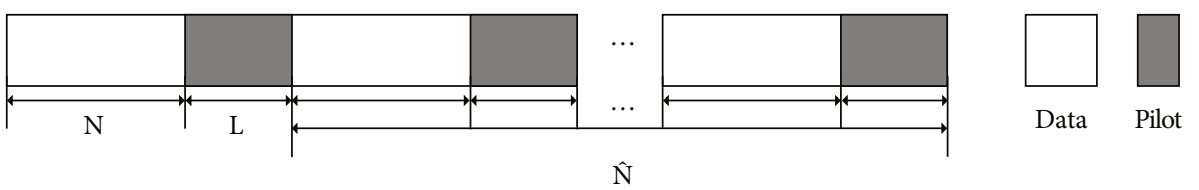

Figure 1: The data-pilot frame structure.

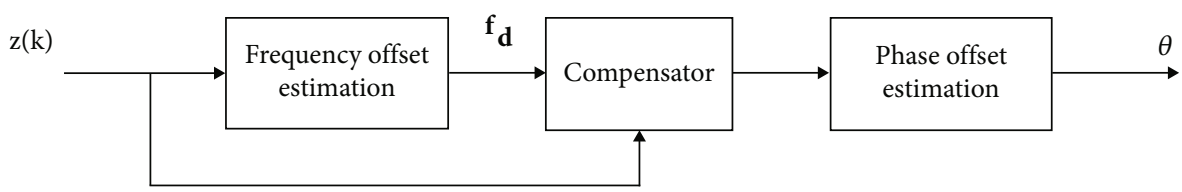

Figure 2: Serial carrier synchronization mode.

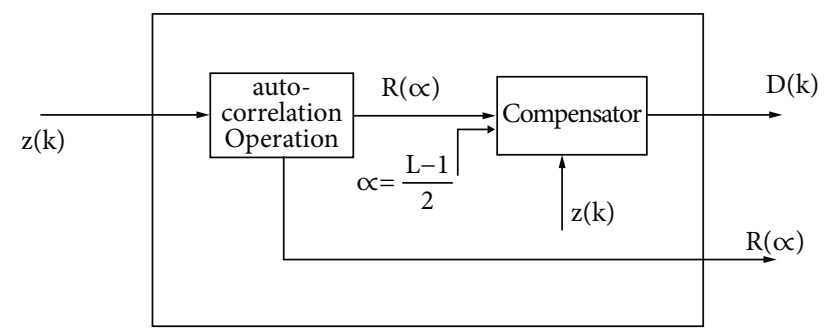

FIGURE 3: The principle block diagram of NCSM.

where $\widehat{\Psi}(k)$ is still the noise interference term. Comparing (9) and (4), each team of $\Psi(\alpha)$ is multiplied by a real number $\lambda_{i}(\alpha)$. We set $\lambda_{i}(\alpha) \leq \lambda_{\max }$, then, let $\Psi_{1}(\alpha)$ be denoted as

$$
\begin{aligned}
\Psi_{1}(\alpha)= & \lambda_{\max } \psi(\alpha)=\frac{1}{L-\alpha} \sum_{k=0}^{L-\alpha-1} \lambda_{\max } e^{-j 2 \pi f_{d} T(k+N)} n(k+\alpha+N) \\
& +\lambda_{\max } e^{j 2 \pi f_{d} T(k+\alpha+N)} n^{*}(k+N)+\lambda_{\max }(\alpha) n(k+\alpha+N) n^{*}(k+N)
\end{aligned}
$$

From (12), we know that $|\Psi(\alpha)| \leq\left|\Psi_{1}(\alpha)\right|$. Because $\mid \psi($ $\alpha) \mid \approx 0$ holds in (4) under high SNR, the value of $|\Psi(\alpha)|$ also approaches zero at this time. By analogy, $|\widehat{\Psi}(k)| \approx 0$ is true when the SNR is high, the result of POE is

$$
\widehat{\theta} \approx \begin{cases}\theta, & N=0 \text { and } \frac{\left|f_{d} T\right| \leq 1}{(L-1)}, \\ H_{2}\left(\theta, f_{d}, N, L\right), & N \neq 0 \text { or } \frac{\left|f_{d} T\right|>1}{(L-1)},\end{cases}
$$

where $H_{2}\left(\theta, f_{d}, N, L\right)$ is still a function representing phase ambiguity. Different from (6), we first calculate the phase angle of $D(k)$ in (10), and then sum $D(k)$. In this way, we can eliminate the influence of signal amplitude information on POE. When $\left|f_{d} T\right|>1 /(L-1)$, the POE performance of (11) is still very poor. The reason is that larger FO results will cause phase folding of $\arg \{D(k)\}$. If we can compensate for the folding phase of $\arg \{D(k)\}$, the POE will not be affected by the pilot overhead. This means that PO estimator can still accurately estimate the PO under a larger FO.

3.1. Phase Unwrapping. In this paper, we use a phase unwrapping method. This method can not only eliminate the phase folding caused by the FO but also greatly reduce the probability of incorrect interval switching caused by the simultaneous presence of noise and FO.

Assume $\vartheta(k)=\widehat{\vartheta}(k)+\Lambda(k)$, where $\widehat{\vartheta}(k)$ is the estimated phase value at time $k, \vartheta(k)$ is the estimated phase value after phase unwrapping, and $\Lambda(k)$ is the phase compensation parameter. The concrete calculation steps are as follows.

(i) Initialization, $\Lambda(0)=0,9(0)=\widehat{\vartheta}(0)$

(ii) $k=k+1$

$$
\Lambda(k)=\left\{\begin{array}{l}
\Lambda(k-1)+2 \pi, \widehat{\vartheta}(k)-\widehat{\vartheta}(k-1)<-\pi, \\
\Lambda(k-1)-2 \pi, \widehat{\vartheta}(k)-\widehat{\vartheta}(k-1)>\pi, \\
\Lambda(k-1), \text { Other } ;
\end{array}\right.
$$

(iii) $\vartheta(k)=\widehat{\vartheta}(k)+\Lambda(k)$

(iv) Repeat step (ii)

The variation of $\Lambda(k)$ is determined by the phase difference between time $k$ and time $k-1$. If the random noise at the moment of $k$ is large, the PO error exceeding $\pi$ will cause $\Lambda(k)$ to change $2 \pi$. If the random noise disappears at $k+k_{0}$ $\left(k_{0} \geq 1\right)$, this will cause the phase estimation difference to exceed $-\pi$ and cause $\Lambda(k)$ to reversely change $2 \pi$. In this case, the carrier phase error will only occur between $k$ and $k_{0}-1$. Therefore, the method is also applicable to the case of low SNR.

3.2. POE Based on ICPEDT. In order to further reduce the influence of FO on POE, phase angles of $z(k)$ and $R(\alpha)$ were 


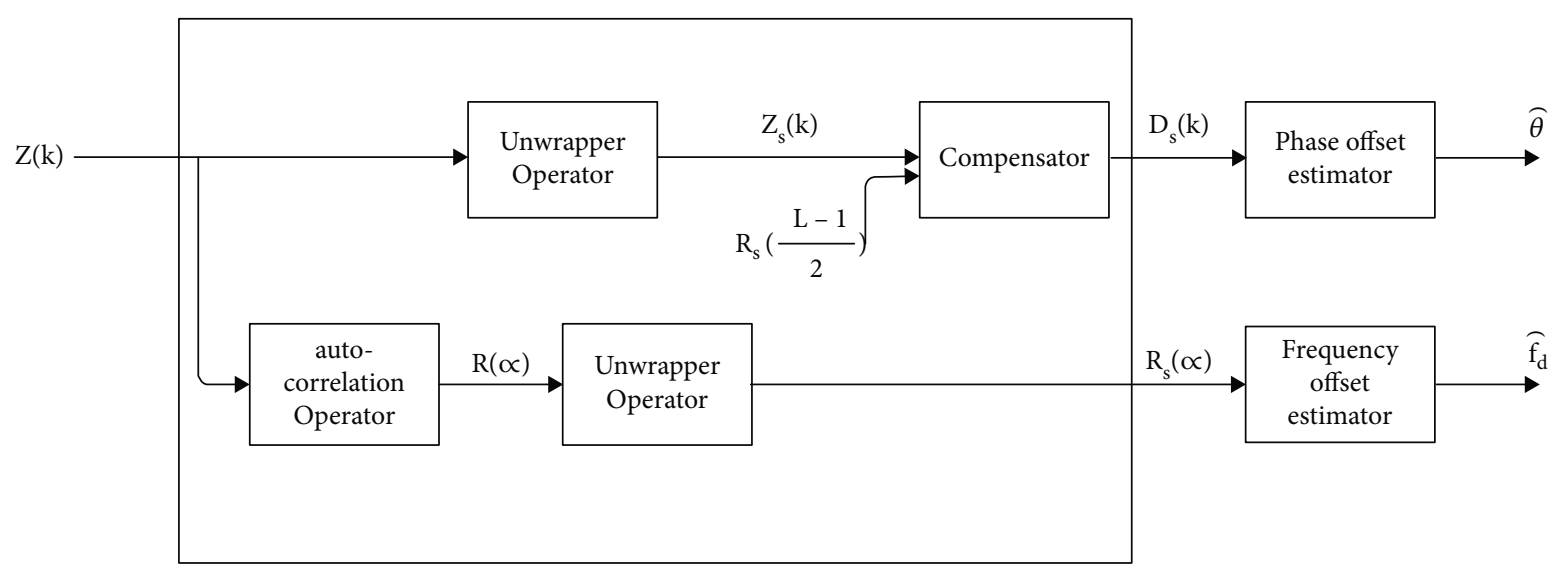

FIGURE 4: The block diagram of ICPEDT.

TABLE 2: Algorithmic complexity comparison of phase offset estimation.

\begin{tabular}{lccc}
\hline & Multiplication & Addition & Complex angle \\
\hline NCSM & $\frac{3 L^{2}+10 L+8}{2}$ & $2 L^{2}+4 L+3$ & $\frac{1}{2} L$ \\
ICPEDT & $\frac{3 L^{2}+18 L}{2}$ & $2 L^{2}+\frac{17}{2} L+1$ & $\frac{3}{2} L$
\end{tabular}

calculated, and phase correction was performed

$$
\begin{gathered}
Z_{s}(k) \triangleq \operatorname{unwrap}\{\arg \{z(k)\}\}, \\
R_{s}(\alpha) \triangleq \operatorname{unwrap}\{\arg \{R(\alpha)\}\} .
\end{gathered}
$$

Then, (11) can be written into

$$
\begin{aligned}
\widehat{\theta}= & \frac{1}{L} \sum_{k=N}^{N+L-1} D_{s}(k)=\frac{1}{L} \sum_{k=N}^{N+L-1} Z_{s}(k)-R_{s}\left(\frac{L-1}{2}\right) \\
= & \frac{1}{L} \sum_{k=N}^{N+L-1} \arg \left\{a_{k} \mathrm{e}^{j\left(2 \pi f_{d} T k+\theta\right)}+d(k)\right\} \\
& -\arg \left\{\gamma_{L-1 / 2} \mathrm{e}^{j 2 \pi f_{d} T L-1 / 2}+\Psi\left(\frac{L-1}{2}\right)\right\} .
\end{aligned}
$$

If the noise interference terms $d(k)$ and $\Psi(L-1 / 2)$ are ignored, we can get

$$
\widehat{\theta} \approx \begin{cases}\theta, & N=0 \\ H_{3}\left(\theta, f_{d}, N\right), & N \neq 0\end{cases}
$$

where $H_{3}\left(\theta, f_{d}, N\right)$ is still a function representing phase ambiguity. From (18), the result of POE is no longer limited by the pilot overhead, so the POE based on ICPEDT has greater antifrequency offset ability after phase unwrapping. At the same time, comparing (11), (17) only needs one real number multiplication operation, so the algorithm complexity is greatly reduced after phase unwrapping. Therefore, the computational complexity of the algorithm in this paper is only a little higher than that of the NCSM. More in detail, the complexity comparison between NCSM and ICPEDT is explained in Table 2.

3.3. FOE Based on ICPEDT. The FO can be estimated directly by the intermediate variable $R_{s}(\alpha) . R_{s}(\alpha)$ is the correction term after phase unwrapping of the autocorrelation operator $R(\alpha)$, and (16) can be expanded as

$$
R_{s}(\alpha)=\operatorname{unwrap}\{\arg \{R(\alpha)\}\}=2 \pi f_{d} T \alpha+\Psi(\alpha)
$$

where $\alpha \in[1, L-1 / 2]$. Sum both ends of (19) separately

$$
\sum_{\alpha=1}^{L-1 / 2} R_{s}(\alpha)=\sum_{\alpha=1}^{L-1 / 2} 2 \pi f_{d} T \alpha+\Psi(\alpha)=\frac{(L-1)(L+1)}{8}\left(2 \pi f_{d} T+\Psi(\alpha)\right) .
$$

If the influence of noise term is ignored, then

$$
\widehat{f}_{d}=\frac{4}{(L-1)(L+1) \pi T} \sum_{\alpha=1}^{L-1 / 2} R_{s}(\alpha) \text {. }
$$

Because the phase of the autocorrelation factor $R_{s}(\alpha)$ is corrected, the phase of $R_{s}(\alpha)$ is the actual FO. Therefore, the estimation range of FOE using $R_{s}(\alpha)$ is no longer affected by the pilot overhead. Theoretically, the frequency offset estimation range is up to $(-0.5,0.5)$.

\section{Simulation and Analysis}

ICPEDT can solve the problem that the FOE directly affects the POE in TCSM and realizes the parallel estimation of FO and PO. According to (18), the selection of the initial position of pilot will affect the accuracy of POE. Therefore, first simulate the influence of the initial position of the pilot on the performance of ICPEDT.

4.1. The Selection of Initial Position $N$ of Pilot. Assume that the modulation mode is QPSK, assume $f_{d} T=0.08$, and $\theta$ $=3 \pi / 8$, the pilot overhead is set to be $L=35$. Figure 5 shows the MSE of POE for different $N$. 


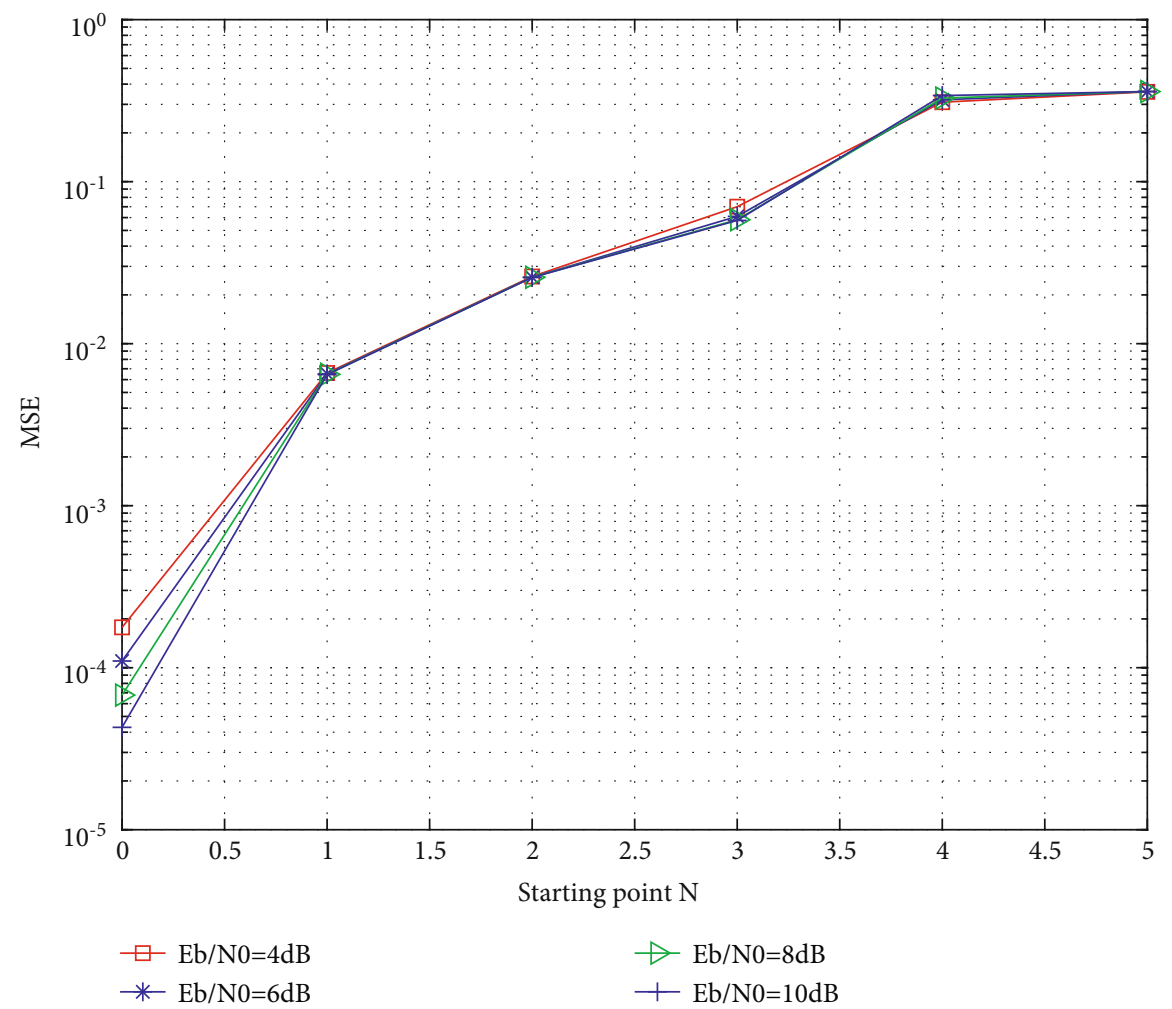

FIgURE 5: The MSE performance of the POE of the ICPEDT at different $N$.

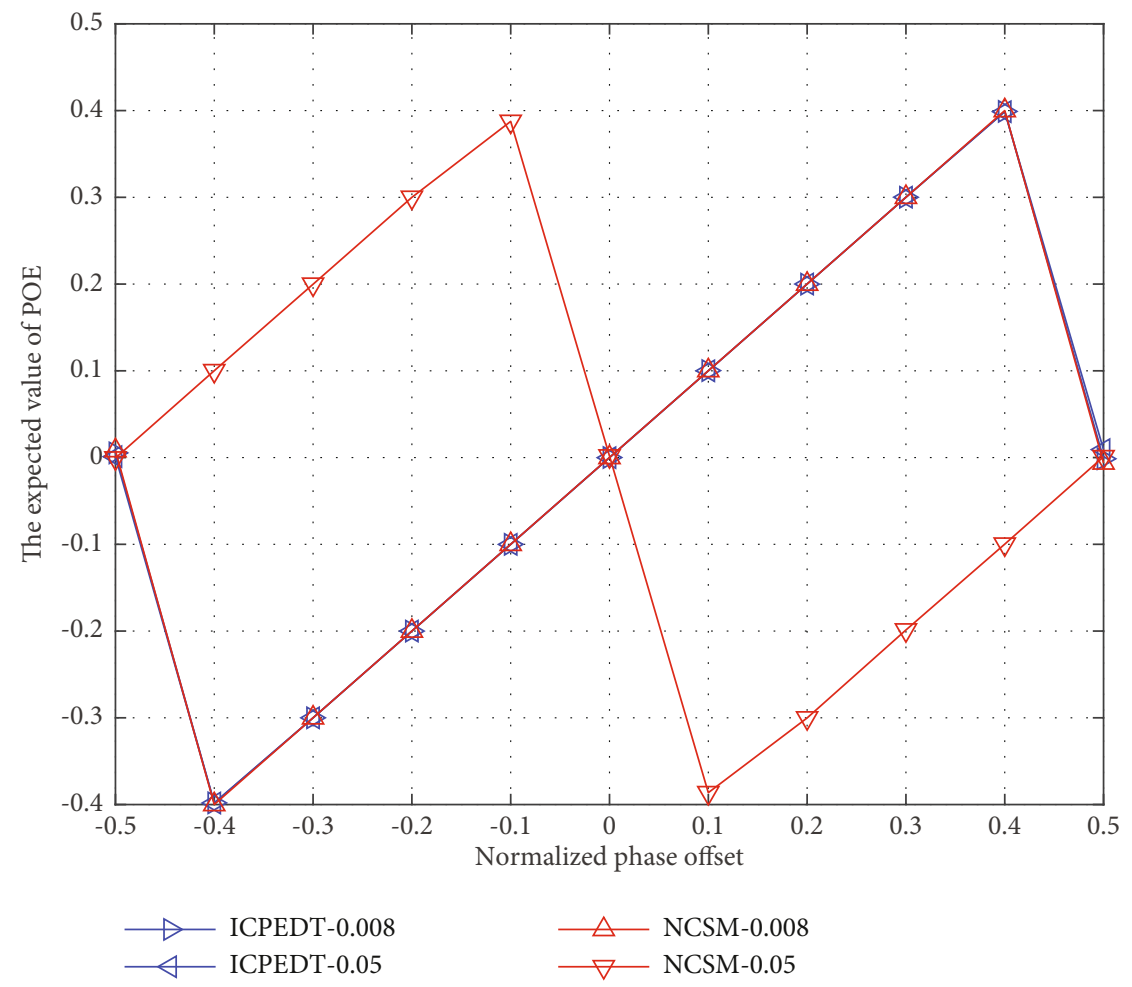

Figure 6: The expectation of the POEs under different POs (QPSK modulation). 


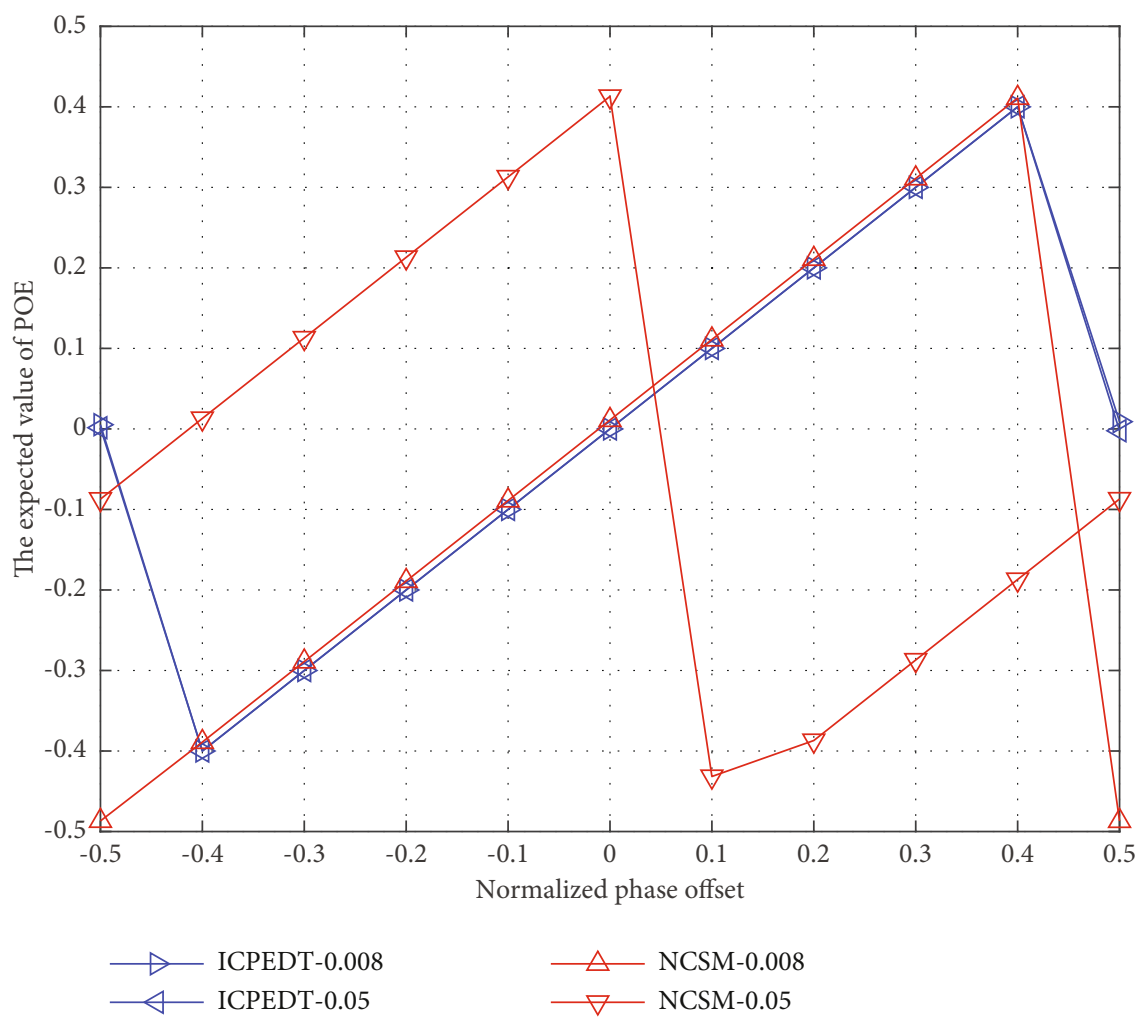

Figure 7: The expectation of the POEs under different POs (32APSK modulation).

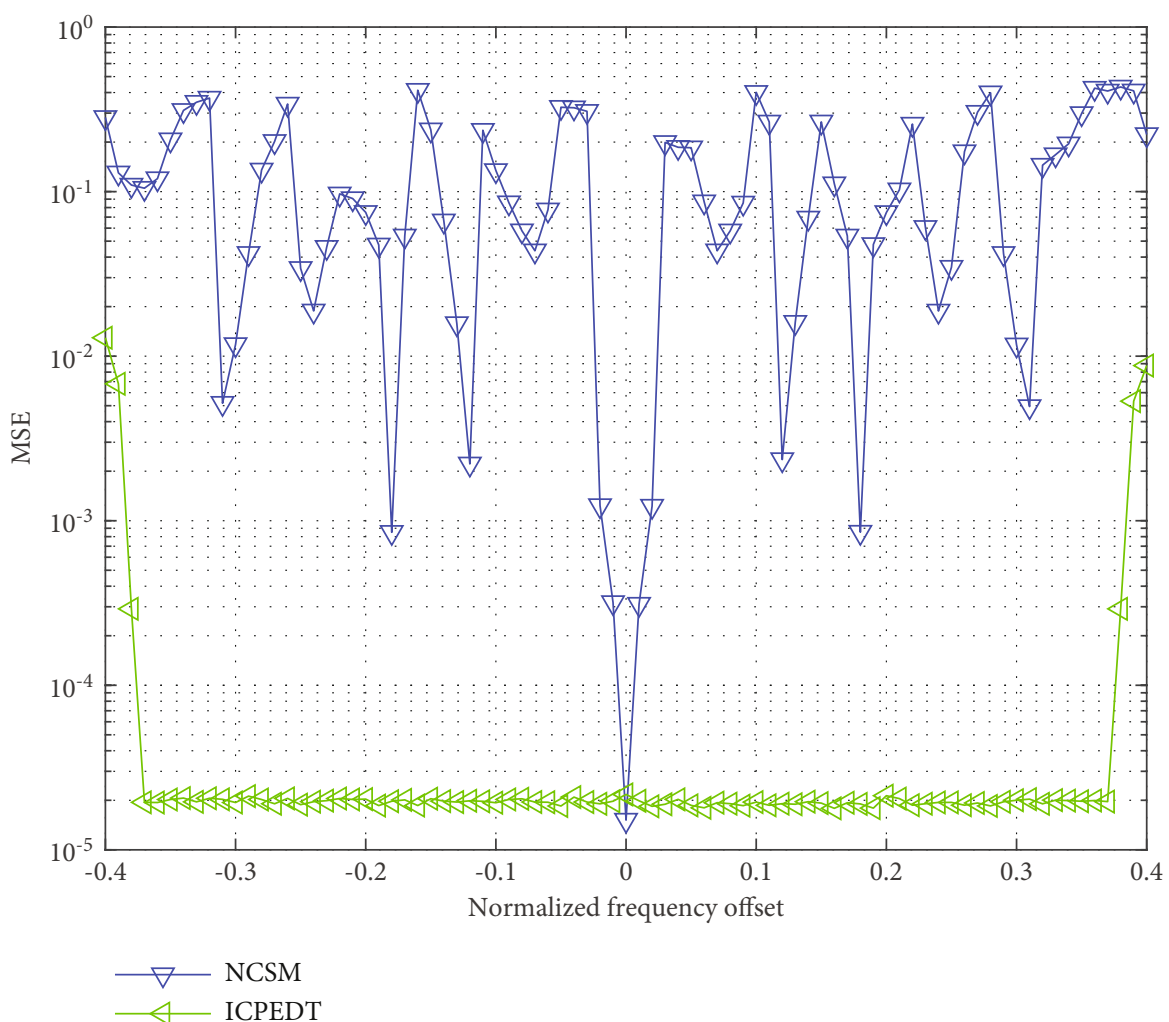

Figure 8: The influence of different FOs on POE. 


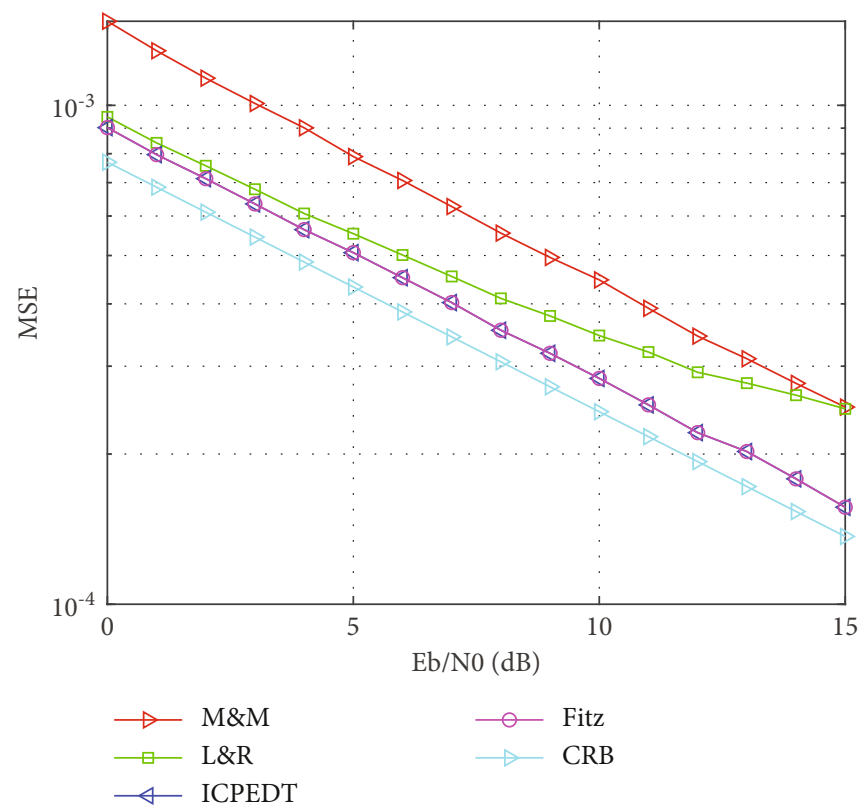

Figure 9: The accuracy of different FOE algorithms.

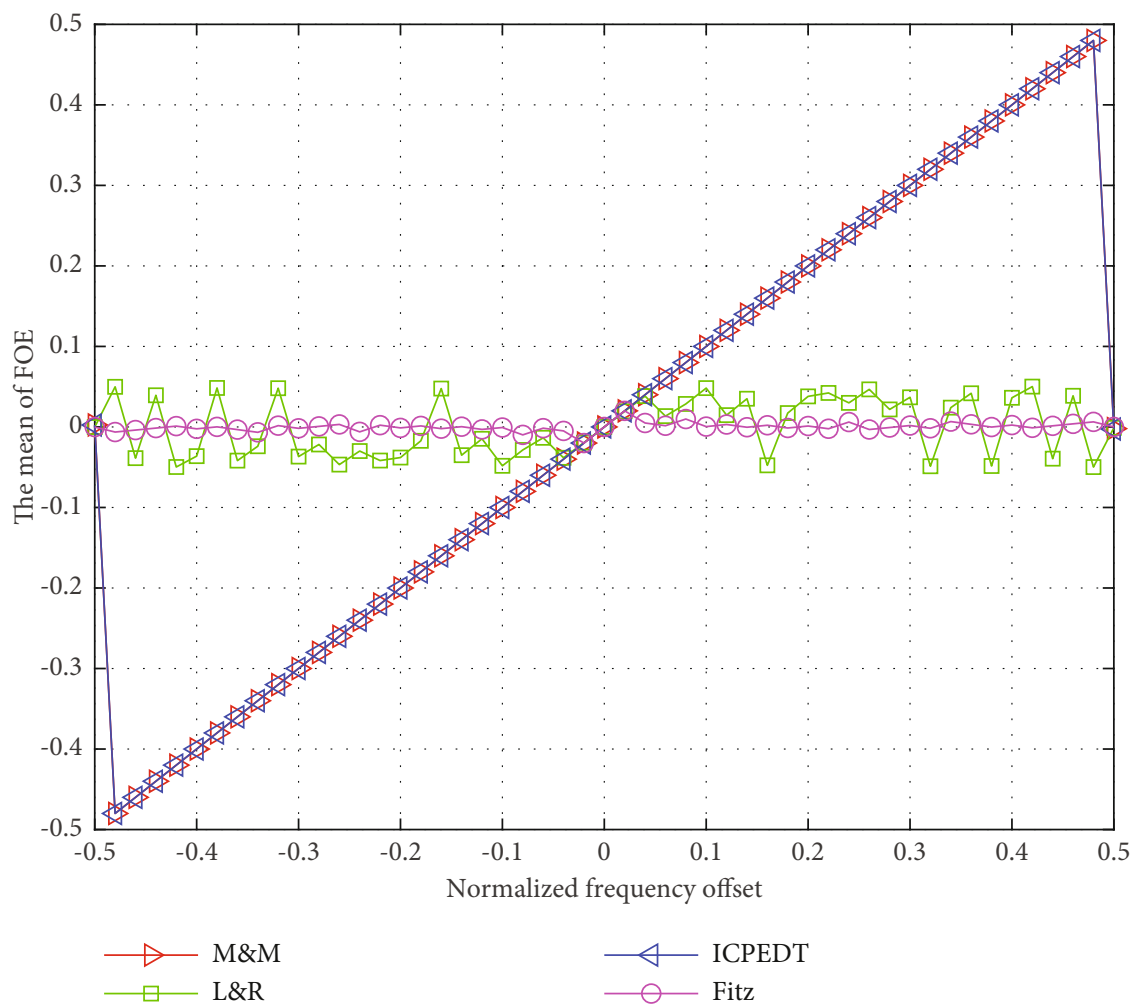

Figure 10: The estimation range of different FOE algorithms.

It can be seen from Figure 5, under different SNR, the POE has the best performance at the initial pilot position $N=0$, and the performance of POE deteriorates with the increase of the initial pilot position. Therefore, in order to obtain the best performance under large FO, the starting point $N$ should be set to zero. In the simulation parameters below, the pilot overhead $L$ is set to 35 and $N=0$.
4.2. The Performance of POE under Different Modulation System. First, assume the modulation system is QPSK, $f_{d} T$ $=0.008,0.05$, the normalized PO $\theta \in(-0.5,0.5], E_{b} / N_{0}=8$ $d B$. Then, set the modulation system to $32 \mathrm{APSK}, E_{b} / N_{0}=$ $15 d B$, and other simulation conditions remain unchanged. Figures 6 and 7 show the expectation of POE of ICPEDT and NCSM for different PO. 
We can see from Figures 6 and 7, in the case of QPSK modulation and the normalized FO is 0.008 , the value of POE of NCSM basically coincides with the actual PO. But when the normalized FO is 0.05 , the result of POE of NCSM has a large deviation from the actual PO. The reason is that NCSM's precise estimation range is limited. It can be seen from (6) that when $L=35$, the POE range of NCSM is $(-0.0286,0.0286)$. So when FO is 0.05 , NCSM's estimation deviation is very large. When the signal is 32APSK, the overall performance of NCSM's POE decreases, and the estimation accuracy deteriorates. However, the estimated value of POE of ICPEDT under different modulations is very close to the actual PO, and the estimation accuracy does not deteriorate with the increase of the frequency deviation, which is consistent with the conclusion of (18).

4.3. The MSE of POE under Different Normalized FO. Assume the modulation is 64APSK, $f_{d} T=[-0.4,0.4], \theta=3$ $\pi / 8$, and $E_{b} / N_{0}=15 d B$, Figure 8 shows the MSE of the POE of ICPEDT and NCSM under different FOs.

It can be seen from the simulation results in Figure 8 that the decoupling performance of NCSM is poor under 64APSK modulation, and the PO can be accurately estimated only when FO is small. However, ICPEDT's POE can still work well under large FO. The method in this paper has strong robust. This is because the phase unwrapping module eliminates the estimation error caused by phase folding, thereby significantly reducing the influence of FOE on the performance of POE.

4.4. The FOE Accuracy of Different Algorithms. Assume the modulation method of the signal is 64APSK, $f_{d} T=0.02$, and $\theta=3 \pi / 8$. Figure 9 shows the estimation accuracy performance curve of different algorithms.

The simulation curves show that the M\&M [3] algorithm has the worst estimate accuracy. The estimation performance of L\&R [4] algorithm gradually deteriorates with the increase of $E_{b} / N_{0}$. The main reason is that the L\&R algorithm does not consider the impact of signal amplitude information on POE. The estimation accuracy of FOE based on ICPEDT is close to the performance of Fitz [5] algorithm, which is better than L\&R and M\&M algorithms. Its performance is close to the lower bound of FOE performance CRB. This shows that the autocorrelation FOE algorithm based on equation (21) is unbiased.

4.5. The FOE Range of Different Algorithms. Assume the simulation signal is 64APSK, $f_{d} T \in(-0.5,0.5], \theta=3 \pi / 8$, and $E_{b} / N_{0}=15 d B$. Figure 10 shows the estimated range of different FOE algorithms.

We can see from Figure 10 that the Fitz algorithm has the smallest estimation range of FO, which is about $(-0.028,0.028)$, followed by the L\&R algorithm. The estimated range of FOE based on ICPEDT is consistent with the estimated range of the M\&M algorithm, which is close to $(-0.5,0.5]$. The simulation results are consistent with the conclusion derived from (21).

\section{Conclusions}

According to the characteristics of satellite communication, we propose a strong robust carrier parameter decoupling technique suitable for high-order APSK modulation. This technique can realize parallel estimation of FO and PO. Both theory and simulation show that, compared with the existing methods, the method in this paper is more robust to the decoupling of high-order APSK modulated signals, and the POE of the method in this paper has better estimation performance and stronger antifrequency offset ability. The accuracy of the FOE in this paper is equivalent to the Fitz method, but it has a larger FOE range. The range of FOE in this paper is close to $(-0.5,0.5]$, but the computational complexity is similar to the Fitz algorithm. Therefore, this method has good engineering practical value.

\section{Data Availability}

No data were used to support this study.

\section{Conflicts of Interest}

The authors declare that there are no conflicts of interest regarding the publication of this paper.

\section{Acknowledgments}

This work is also supported in part by the National Key Research and Development Program under Grant 2019QY0302, in part by the Key Young Teachers Project of Henan Province under Grant 2018GGJS006, and in part by the Key Scientific Research Project of Colleges and Universities in Henan Province under Grant 20A510010 and Grant 20A510007.

\section{References}

[1] X. Li, M. Zhao, M. Zeng et al., "Hardware impaired ambient backscatter NOMA systems: reliability and security," IEEE Transactions on Communications, vol. 69, no. 4, pp. 2723 2736, 2021.

[2] W. U. Khan, M. A. Javed, T. N. Nguyen, S. Khan, and B. M. Elhalawany, "Energy-efficient resource allocation for 6G backscatter-enabled NOMA IoV networks," IEEE Transactions on Intelligent Transportation Systems, pp. 1-11, 2021.

[3] J. Xia, D. Deng, and D. Fan, "A note on implementation methodologies of deep learning-based signal detection for conventional MIMO transmitters," IEEE Transactions on Broadcasting, vol. 66, no. 3, pp. 744-745, 2020.

[4] K. Omprakash and K. Sushil, "Geocast routing: recent advances and future challenges in vehicular adhoc networks," 2014 International Conference on Signal Processing and Integrated Networks, SPIN, 2014, pp. 291-296, Noida, India, 2014.

[5] A. Sikandar, R. Agrawal, M. K. Tyagi, A. L. N. Rao, M. Prasad, and M. Binsawad, "Toward green computing in wireless sensor networks: prediction-oriented distributed clustering for non-uniform node distribution," Journal on Wireless Communications and Networking, vol. 2020, no. 1, p. 183, 2020.

[6] ETSI E, Digital Video Broadcasting (DVB): Second Generation Framing Structure, Channel Coding and Modulation Systems 
for Broadcasting, Interactive Services, New Gathering and Other Broadband Satellite Applications, Part2:DVB-S2 Extensions(DVB-S2X), 2014.

[7] X. Li, H. Mengyan, Y. Liu, V. G. Menon, A. Paul, and Z. Ding, "I/Q imbalance aware nonlinear wireless-powered relaying of B5G networks: security and reliability analysis," IEEE Transactions on Network Science and Engineering, vol. 8, no. 4, pp. 2995-3008, 2021.

[8] W. U. Khan, F. Jameel, X. Li, M. Bilal, and T. A. Tsiftsis, "Joint spectrum and energy optimization of NOMA-enabled smallcell networks with QoS guarantee," IEEE Transactions on Vehicular Technology, vol. 70, no. 8, pp. 8337-8342, 2021.

[9] U. Mengali and M. Morelli, "Data-aided frequency estimation for burst digital transmission," IEEE Transactions on Communications, vol. 45, no. 1, pp. 23-25, 1997.

[10] M. Luise and R. Reggiannini, "Carrier frequency recovery in all-digital modems for burst-mode transmissions," IEEE Transactions on Communications, vol. 43, no. 2/3/4, pp. 1169-1178, 1995.

[11] M. Fitz, "Further results in the fast estimation of a single frequency," IEEE Transactions on Communications, vol. 42, no. 2/3/4, pp. 862-864, 1994.

[12] N. Noels, C. Herzet, A. Dejonghe et al., "Turbo synchronization: an EM algorithm interpretation," in IEEE International Conference on Communications, 2003. ICC '03, vol. 4, pp. 2933-2937, Anchorage, AK, USA, 2003.

[13] N. Noels, V. Lottici, A. Dejonghe et al., "A theoretical framework for soft-information-based synchronization in iterative (turbo) receivers," EURASIP Journal on Wireless Communications and Networking, vol. 2005, no. 1, 2005.

[14] M. Zhengke, Z. Haoliang, W. Wei, and Z. Shenghua, "A simplified code-aided carrier synchronization algorithm," in 2019 2nd International Conference on Safety Produce Informatization (IICSPI), pp. 283-286, Chongqing, China, 2019.

[15] C. Wang, Y. Li, and K. Li, “An high-precision FFT frequency offset estimation algorithm based on interpolation and binary search," in 2019 IEEE 3rd information technology, networking, Electronic and Automation Control Conference (ITNEC), pp. 437-442, Chengdu, China, 2019.

[16] T. Li, Y. Zhang, and B. Tang, "An improved DFT frequency offset estimation algorithm with high accuracy," in 2019 IEEE 3rd information technology, networking, Electronic and Automation Control Conference (ITNEC), pp. 175-179, Chengdu, China, 2019.

[17] J. Fan, Y. Ren, X. Luo, and J. Joung, "Iterative carrier frequency offset estimation scheme for faster-than-Nyquist signaling systems," IEEE Photonics Technology Letters, vol. 32, no. 18, pp. 1203-1206, 2020.

[18] Z. Yong-gang, Y. Fu-qiang, L. Yong-xiang, Z. Yi, and P. Wei, "ML-based carrier frequency offset estimation and its low SNR threshold," Systems Engineering and Electronics, vol. 33, pp. 427-431, 2011.

[19] Z. Yu, J. Sun, B. Bai, and X. Wu, "A phase increment-based frequency estimator for general psam in burst communications," in 2016 IEEE 83rd Vehicular Technology Conference (VTC Spring), pp. 1-5, Nanjing, China, 2016.

[20] F. Rice, "Carrier-phase and frequency-estimation bounds for transmissions with embedded reference symbols," IEEE Transactions on Communications, vol. 54, no. 2, pp. 221-225, 2006.
[21] N. Noels, H. Steendam, M. Moeneclaey, and H. Bruneel, "Carrier phase and frequency estimation for pilot-symbol assisted transmission: bounds and algorithms," IEEE Transactions on Signal Processing, vol. 53, no. 12, pp. 4578-4587, 2005.

[22] H. Xu, Z. Yu, H. Zhu, M. Xu, and Z. Luo, "Frequency-phase decoupling method for carrier synchronization in space communications," IEEE Wireless Communications Letters, vol. 8, no. 4, pp. 1293-1296, 2019. 